\title{
Soluble TRAIL is present at high concentrations in seminal plasma and promotes spermatozoa survival
}

\author{
Giorgio Zauli*, Claudio Celeghini ${ }^{1, *}$, Lorenzo Monasta, Monica Martinelli, Stefania Luppi, \\ Arianna Gonelli ${ }^{2}$, Vittorio Grill ${ }^{1}$, Giuseppe Ricci and Paola Secchiero ${ }^{2}$ \\ Institute for Maternal and Child Health, IRCCS 'Burlo Garofolo', Via dell'Istria 65/1, 34137 Trieste, Italy, \\ ${ }^{1}$ Department of Life Science, University of Trieste, 34128 Trieste, Italy and ${ }^{2}$ Department of Morphology, \\ Surgery and Experimental Medicine, LTTA Centre, University of Ferrara, 44100 Ferrara, Italy \\ Correspondence should be addressed to G Zauli; Email: giorgio.zauli@unife.it \\ *(G Zauli and C Celeghini contributed equally to this work)
}

\begin{abstract}
The expression of tumor necrosis factor-related apoptosis-inducing ligand (TRAIL(TNFSF10)) and of its receptors (TRAILR1, TRAILR2, TRAILR3, and TRAILR4) have been documented in testis, but the presence of soluble TRAIL in seminal fluid, as well as the potential physiopathological role of the TRAIL/TRAILR system in spermatozoa, has not been previously investigated. Male donors ( $n=123$ ) among couples presenting for infertility evaluation were consecutively enrolled in this study. The presence of soluble TRAIL was analyzed in seminal samples by ELISA, while the surface expression of TRAIL receptors was investigated by flow cytometry. High levels of soluble TRAIL were detected in seminal plasma (median, $11621 \mathrm{pg} / \mathrm{ml}$ and mean \pm s.D., $13371 \pm 8367 \mathrm{pg} / \mathrm{ml}$ ) and flow cytometric analysis revealed a variable expression of TRAIL receptors in the sperm cellular fraction among different subjects. In addition, the effect of physiologically relevant concentrations of recombinant TRAIL was investigated on survival and motility of spermatozoa. Of interest, the in vitro exposure of capacitated spermatozoa to recombinant TRAIL $(10 \mathrm{ng} / \mathrm{ml})$ significantly preserved their overall survival. Therefore, the present study demonstrates for the first time the presence of elevated levels of the anti-inflammatory cytokine TRAIL in seminal fluids. Moreover, the demonstration that recombinant TRAIL promotes spermatozoa survival after capacitation suggests potential therapeutic implications.
\end{abstract}

Reproduction (2014) 148 191-198

\section{Introduction}

Tumor necrosis factor-related apoptosis-inducing ligand (TRAIL(TNFSF10)) is expressed as a type 2 membrane protein or as a soluble cytokine (Secchiero \& Zauli 2008), and it is present in detectable amounts in the plasma/ serum as well as in other body fluids of normal individuals (Secchiero et al. 2009, 2010, Bernardi et al. 2012). TRAIL acts as a homotrimer, interacting with any one of five cognate receptors, four transmembrane (TRAILR1, TRAILR2, TRAILR3, and TRAILR4), and one soluble (osteoprotegerin (OPG)) receptor, albeit with different affinities (Zauli et al. 2007, 2009). The best-characterized biological function of the two TRAIL-receptors containing a death-domain (TRAILR1 and TRAILR2) is to elicit an apoptotic response upon binding of TRAIL. However, in a series of studies, we have demonstrated that alternative TRAIL signaling is unmasked in cells resistant to TRAIL-induced apoptosis, in which the predominant result of TRAILR2 engagement is the activation of intracellular signal transduction pathways, such as
ERK/MAPK, AKT, and NF-KB (Secchiero et al. 2001, 2008, Milani et al. 2003, Zauli et al. 2005, 2008). TRAILR3 and TRAILR4 are considered as membrane neutralizing or regulatory receptors. Although OPG has been initially identified as a member of the soluble tumor necrosis factor (TNF)-receptor family, inhibiting RANKLmediated osteoclastogenesis, several in vitro studies have shown its ability to also neutralize TRAIL (Emery et al. 1998, Miyashita et al. 2004, Zauli et al. 2009).

Among different tissues, it is noteworthy that TRAIL and its receptors are abundantly expressed in human testis (Grataroli et al. 2004). Although TRAIL has been proposed to contribute to the control of the number of spermatogonia (Coureuil et al. 2010), the physiopathological role of TRAIL in testis remains to be fully elucidated. In addition, the presence of TRAIL in seminal plasma has never been evaluated. It should also be taken into consideration that a growing number of studies from different groups of investigators have shown that TRAIL displays anti-inflammatory activity (Li et al. 2012, Marcuzzi et al. 2012, Keuper et al. 2013, 
Table 1 Demographics and descriptive analysis of semen parameters of male subjects $(n=90)$.

\begin{tabular}{lc}
\hline Variables & $\begin{array}{c}\text { Mean (s.D.) } \\
\text { or median (IQR) }\end{array}$ \\
\hline Age (years) & $39.7(5.5)$ \\
BMI & $24.9(23.1-27.5)$ \\
Semen parameters & \\
Sperm concentration (million/ml) & $35(15-85)$ \\
Rapid progressive sperm motility (A, \%) & $15(5-20)$ \\
Slow progressive sperm motility (B, \%) & $15(10-25)$ \\
Morphology (\% normal form) & $22(18-26)$ \\
Leucocytes in semen (million/ml) & $0.03(0.00-0.36)$ \\
Male etiology of infertility (no. of cases) & \\
Negatives & 54 \\
Varicocele & 21 \\
Cryptorchidism & 7 \\
Testicular hypotrophy & 3 \\
Erectile dysfunction & 2 \\
Epididymal cyst & 1 \\
Hematospermia & 1 \\
Testicular hydrocele & 1
\end{tabular}

IQR, interquartile range; WHO, World Health Organization. ${ }^{a}$ Mean ( \pm s.D.) are reported for variable distributing normally according to skewness and kurtosis joint test; medians and IQR are reported otherwise. ${ }^{\text {b}}$ Semen quality parameters according to the WHO.

Secchiero et al. 2013, Walczak 2013). On these bases, the aim of our present study was i) to analyze the presence and the levels of soluble TRAIL in the seminal samples and the surface expression of TRAIL receptors in the sperm cellular fraction and ii) to evaluate the potential biological activity of soluble TRAIL, by assessing the in vitro response of spermatozoa in terms of vitality/survival and motility upon exposure to recombinant TRAIL.

\section{Materials and methods}

\section{Subjects and semen collection}

The study has been conducted according to the principles expressed in the Declaration of Helsinki. Institutional Review Board approval was obtained from the Internal Ethical Committee of the Institute for Maternal and Child Health, IRCCS 'Burlo Garofolo' (Trieste), that authorized the use of semen samples submitted to semen examination for experimental purposes. Each donor gave informed consent allowing the use of his semen for our study. Sperm samples were obtained by masturbation after $4-5$ days of sexual abstinence from a total of 123 male donors among couples presenting for infertility evaluation. All samples were allowed to liquefy for at least $30 \mathrm{~min}$ at $37^{\circ} \mathrm{C}$, and then were evaluated for sperm concentration, vitality, motility, and morphology according to the guidelines of World Health Organization (WHO). Motile spermatozoa were then capacitated by the swim-up technique $\left(37^{\circ} \mathrm{C}\right.$ for $75 \mathrm{~min}$ in a $5 \% \mathrm{CO}_{2}$ atmosphere) using sperm washing medium (SWM), as described previously (Miraglia et al. 2010). After swim-up, the motile sperm-rich fraction was centrifuged at $600 \mathrm{~g}$ for $10 \mathrm{~min}$, the supernatant was discarded, and the pellets were re-suspended in SWM before evaluation for sperm concentration, vitality, motility, and morphology according to the guidelines of $\mathrm{WHO}$.

\section{Measurement of soluble TRAIL}

Seminal plasma was collected from liquefied semen samples by centrifugation at $1000 \mathrm{~g}$ for $10 \mathrm{~min}$, followed by a second centrifugation of the supernatants at $16000 \mathrm{~g}$ for $15 \mathrm{~min}$ to remove debris and insoluble components. After centrifugations, the seminal plasma samples were collected and stored in aliquots at $-80^{\circ} \mathrm{C}$. In selected cases $(n=10)$, TRAIL was measured in both plasma and cellular fractions of the same semen samples. Analysis of TRAIL levels was carried out by using specific, commercially available ELISA Kit (R\&D Systems, Minneapolis, MN, USA) in accordance with the manufacturer's instructions and as previously described (Secchiero et al. 2009, 2010). Sensitivity of the assay was $2.86 \mathrm{pg} / \mathrm{ml}$ with the intra- and inter-assay coefficients of variation being 3.9 and $6 \%$
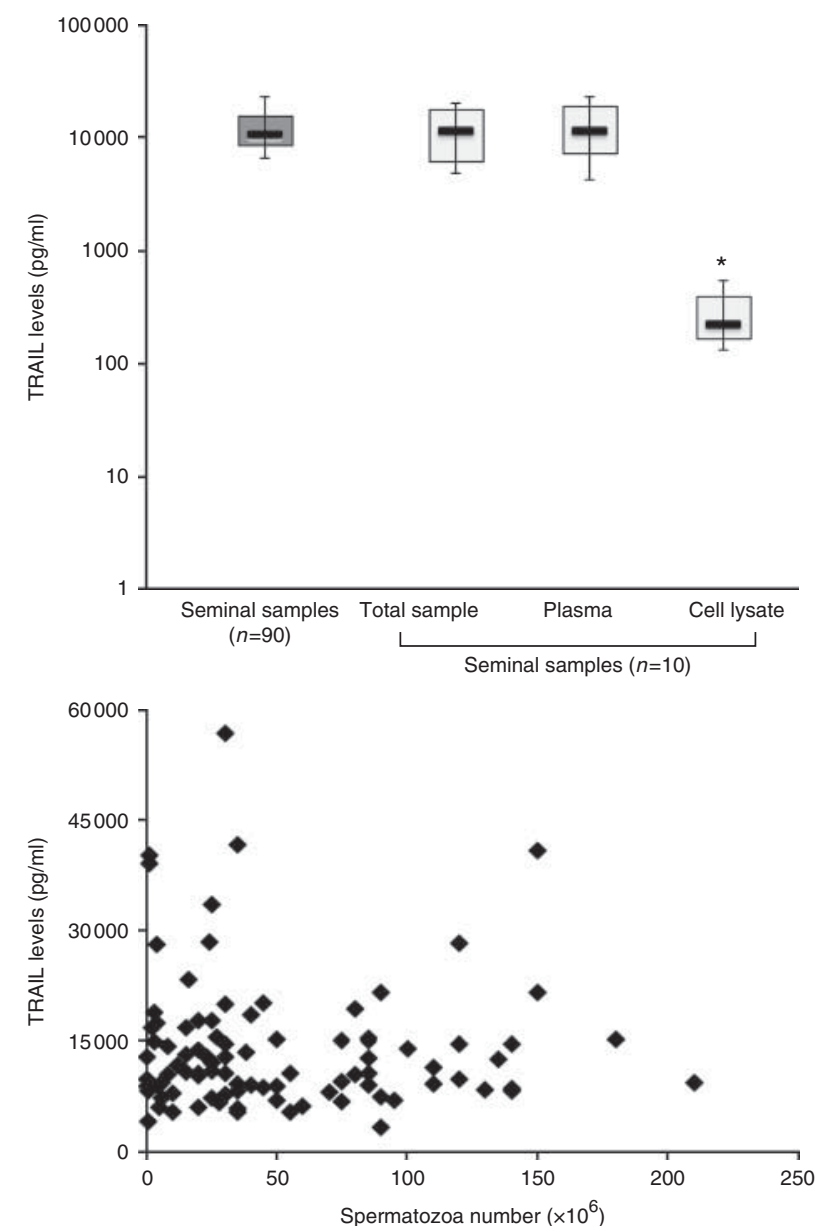

Figure 1 Soluble TRAIL levels in seminal samples. Levels of soluble TRAIL were measured in seminal samples by ELISA. (A) TRAIL levels were assessed in 90 seminal samples. In ten samples, TRAIL levels were comparatively determined in the unfractionated (total) sample, plasma, and cell lysate. Horizontal bars are median, upper and lower edges of box are 75 th and 25 th percentiles, lines extending from box are 10th and 90th percentiles. ${ }^{*} P<0.01$ compared with the levels measured in the seminal plasma and total seminal samples. (B) Lack of significant correlation between the seminal levels of TRAIL and the number of spermatozoa. Correlation coefficient $(R)$, calculated by Spearman's analysis, and $P$ value are reported in the text. 
A
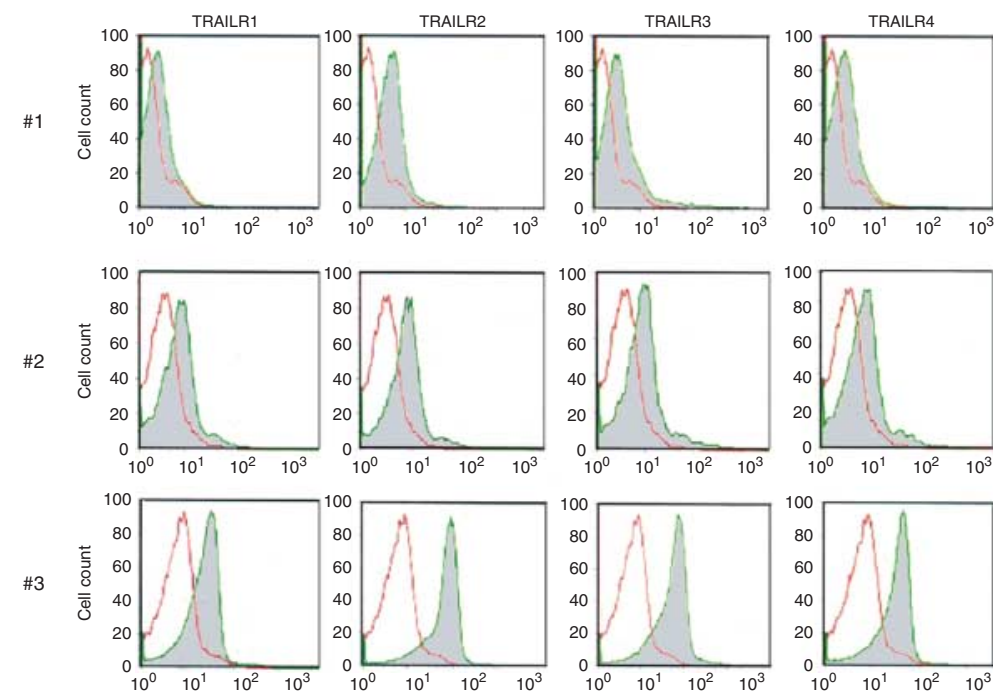

- Surface expression (relative fluorescence)
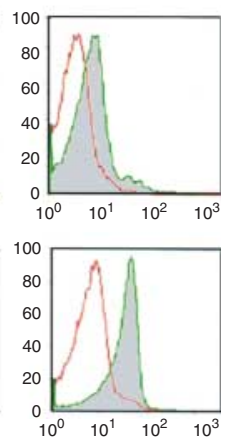

B
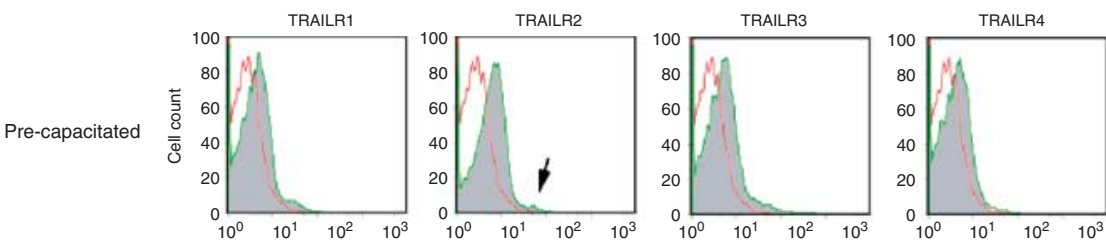

Capacitated
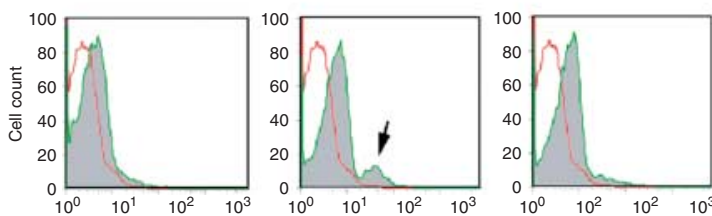

- Surface expression (relative fluorescence) -

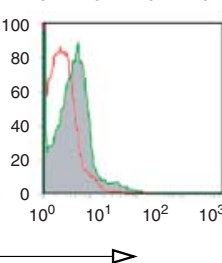

Figure 2 Surface expression of TRAIL receptors in spermatozoa. Surface expression of TRAILR1, TRAILR2, TRAILR3, and TRAILR4 was determined by flow cytometric analysis of seminal samples. Shadowed histograms represent cells stained with mAbs specific for the indicated surface antigens, while unshadowed histograms represent the background fluorescence obtained from the staining of the same samples with isotype-matched control mAbs. (A) Representative phenotypes of seminal samples analyzed before capacitation are shown. (B) Comparison of the TRAILR phenotype of the seminal samples before and after capacitation, showing the enrichment of a subpopulation (arrow) with higher TRAILR2 expression upon capacitation. Data are representative of three different cases. respectively and the upper limit of detection being $1000 \mathrm{pg} / \mathrm{ml}$. Serial dilutions (1:5) of each sample were run in duplicate. Some samples were run in each ELISA plates, as internal controls, confirming the reproducibility of the determinations over times.

\section{Phenotypic analysis and assessment of sperm vitality, apoptosis, and motility}

Surface TRAIL receptor expression in both pre- and postcapacitated samples was analyzed by using PE-conjugated mAbs anti-human TRAILR1 (FAB347P), TRAILR2 (FAB6311P), TRAILR3 (FAB6302P), and TRAILR4 (FAB633P; all from R\&D Systems), as described previously (Rimondi et al. 2006). Assessment of sperm vitality was carried out by using the one-step eosin-nigrosin staining technique as previously reported (Björndahl et al. 2003) and according to the WHO guidelines of 2010. In parallel, in capacitates samples, assessment of apoptosis was evaluated by double staining with PI and FITC-conjugated Annexin V (Alexis Biochemical, Lausen, Switzerland) and flow cytometry analysis as previously detailed (Re et al. 1993, Zauli et al. 1995, Vitale et al. 1997).
The sperm motility parameters were assessed by CASA (CGAWLJY-9000; CGA Distribution, Florence, Italy; Krause \& Viethen 1999). The following kinetic parameters were determined: percentage of spermatozoa exhibiting a forward progressive motility (A and B WHO classes), in situ motility (C WHO class), or no motility (D WHO class). For the analysis of the potential effects of TRAIL on spermatozoa vitality and motility, aliquots of sperm suspension $(200 \mu \mathrm{l})$ were incubated in the absence or presence of recombinant TRAIL (used in the range of $1-10 \mathrm{ng} / \mathrm{ml}$ ), prepared as described previously (Milani et al. 2003, Zauli et al. 2008). For specificity assays, sperm samples were treated with either recombinant human OPG (R\&D Systems; $10 \mathrm{ng} / \mathrm{ml}$ ) or recombinant TRAIL pre-incubated with OPG used at the same $(1: 1)$ concentration.

\section{Statistical analysis}

Box plots were used to show the median, interquartile ranges, and minimum and maximum values for each group of data. After verifying that TRAIL values of this study did not distribute normally (skewness and kurtosis joint normality test), 
Table 2 Semen variables in samples employed for the in vitro assays in response to recombinant TRAIL.

\begin{tabular}{|c|c|c|c|c|c|c|c|c|c|c|c|}
\hline \multirow{3}{*}{$\begin{array}{l}\text { Subject } \\
\text { no. }\end{array}$} & \multicolumn{6}{|c|}{ Pre-capacitated } & \multicolumn{5}{|c|}{ Capacitated } \\
\hline & \multirow{2}{*}{$\begin{array}{l}\text { Volume } \\
(\mathrm{ml})\end{array}$} & \multirow{2}{*}{$\begin{array}{c}\text { Sperm concen- } \\
\text { tration }\left(\times 10^{6} / \mathrm{ml}\right)\end{array}$} & \multicolumn{4}{|c|}{ Motility WHO classes (\%) } & \multirow{2}{*}{$\begin{array}{c}\text { Sperm concen- } \\
\text { tration }\left(\times 10^{6} / \mathrm{ml}\right)\end{array}$} & \multicolumn{4}{|c|}{ Motility WHO classes (\%) } \\
\hline & & & A & $\mathrm{B}$ & $\mathrm{C}$ & $\mathrm{D}$ & & A & $\mathrm{B}$ & $\mathrm{C}$ & $\mathrm{D}$ \\
\hline 101 & 3 & 120 & 30 & 30 & 20 & 20 & 65 & 80 & 15 & 5 & 0 \\
\hline 102 & 5.6 & 12 & 10 & 10 & 30 & 40 & 0.6 & 70 & 20 & 10 & 0 \\
\hline 103 & 3 & 80 & 19 & 14 & 7 & 60 & 2.5 & 64 & 26 & 10 & 0 \\
\hline 104 & 3.5 & 26 & 10 & 34 & 14 & 42 & 3 & 80 & 10 & 20 & 0 \\
\hline 105 & 5 & 80 & 15 & 35 & 20 & 30 & 1.4 & 90 & 10 & 0 & 0 \\
\hline 106 & 2.7 & 65 & 10 & 15 & 25 & 50 & 3 & 100 & 0 & 0 & 0 \\
\hline 107 & 3.6 & 135 & 17 & 44 & 17 & 22 & 75 & 100 & 0 & 0 & 0 \\
\hline 108 & 2.5 & 150 & 20 & 30 & 10 & 40 & 30 & 90 & 10 & 0 & 0 \\
\hline 109 & 2.1 & 55 & 27 & 30 & 22 & 21 & 10 & 100 & 0 & 0 & 0 \\
\hline 110 & 3 & 60 & 25 & 25 & 15 & 35 & 45 & 100 & 0 & 0 & 0 \\
\hline 111 & 5 & 45 & 22 & 22 & 11 & 45 & 5 & 80 & 20 & 0 & 0 \\
\hline 112 & 3.7 & 35 & 26 & 20 & 24 & 30 & 7 & 90 & 10 & 0 & 0 \\
\hline
\end{tabular}

we applied the nonparametric Mann-Whitney $U$ or the Kruskal-Wallis tests for the equality of populations to compare the TRAIL values among different populations. Correlation coefficients were calculated with the Spearman's rank coefficient $\rho$. $P$ value $<0.05$ was considered statistically significant.

\section{Results \\ Presence of high levels of soluble TRAIL in seminal samples}

Although previous studies have documented the presence of TRAIL in the testis (Grataroli et al. 2004, Coureuil et al. 2010), no studies have addressed the presence of TRAIL in semen. Therefore, in the first group of experiments, we have analyzed the presence and levels of TRAIL in a large group of seminal samples $(n=90$; Table 1). As shown in Fig. 1A, concentration of soluble TRAIL in semen samples of donors, among couples presenting for infertility evaluation, was very high in all individuals, showing a median of $10924 \mathrm{pg} / \mathrm{ml}$ (mean \pm s.D., $14109 \pm 9334 \mathrm{pg} / \mathrm{ml}$ ). The levels of TRAIL did not correlate (Sperman's $\rho=-0.0334, P=0.75$ ) with the number of spermatozoa (Fig. 1B). In additional experiments, to evaluate whether TRAIL was associated to the liquid and/or to the cellular components of the seminal samples, we have comparatively analyzed the levels of TRAIL in the seminal plasma and in the corresponding cellular fraction of a subset of samples $(n=10)$. While the seminal plasma showed levels of TRAIL comparable (median, 11621 and mean \pm s.D., $13371 \pm 8367)$ to those of unfractionated samples (median, 11551 and mean \pm s.D., $11995 \pm 6955$ ), the amount of TRAIL associated to the cells was markedly lower (median, 221 and mean \pm s.D., $302 \pm 201$ ) (Fig. 1A). Taken together, these data clearly indicate that most of the soluble TRAIL was present in the liquid fraction rather than being associated to the spermatozoa.

\section{Variable expression of surface TRAIL receptors in human spermatozoa}

In order to start the investigation about the potential significance of high levels of TRAIL in seminal plasma, we have assessed the surface expression of TRAIL receptors (TRAILR1, TRAILR2, TRAILR3, and TRAILR4) in spermatozoa. As exemplified in Fig. 2A, a variable expression pattern was observed among the different samples analyzed. It should be noticed that between the two TRAIL receptors able to transduce both apoptotic and nonapoptotic intracellular signals (TRAILR1 and TRAILR2), TRAILR2 appeared usually at higher expression levels than TRAILR1. Moreover, while TRAILR1, TRAILR3, and TRAILR4 expression did not show appreciable differences before and after capacitation, in some samples analyzed, TRAILR2 expression increased after capacitation, suggesting the enrichment of cell subpopulations exhibiting higher expression (Fig. 2B).

\section{Exposure to recombinant TRAIL increased spermatozoa survival after capacitation}

As TRAIL is known to affect cell survival/apoptosis in a cell type-dependent manner (Bernardi et al. 2012), we have then investigated the effect of physiologically relevant $(1-10 \mathrm{ng} / \mathrm{ml})$ concentrations of recombinant TRAIL on spermatozoa survival/apoptosis. For this purpose, fresh selected samples, with baseline characteristics reported in Table 2, were exposed in vitro to recombinant TRAIL. After 24-48 h of culture, pre-capacitated spermatozoa were virtually all immotile or dead/apoptotic irrespectively of the presence of recombinant TRAIL (data not shown). On the other hand, treatment with recombinant TRAIL significantly preserved the vitality of post-capacitated spermatozoa both at 24 and $48 \mathrm{~h}$ (Fig. 3A). The specificity of the effect was documented in three additional fresh sperm 

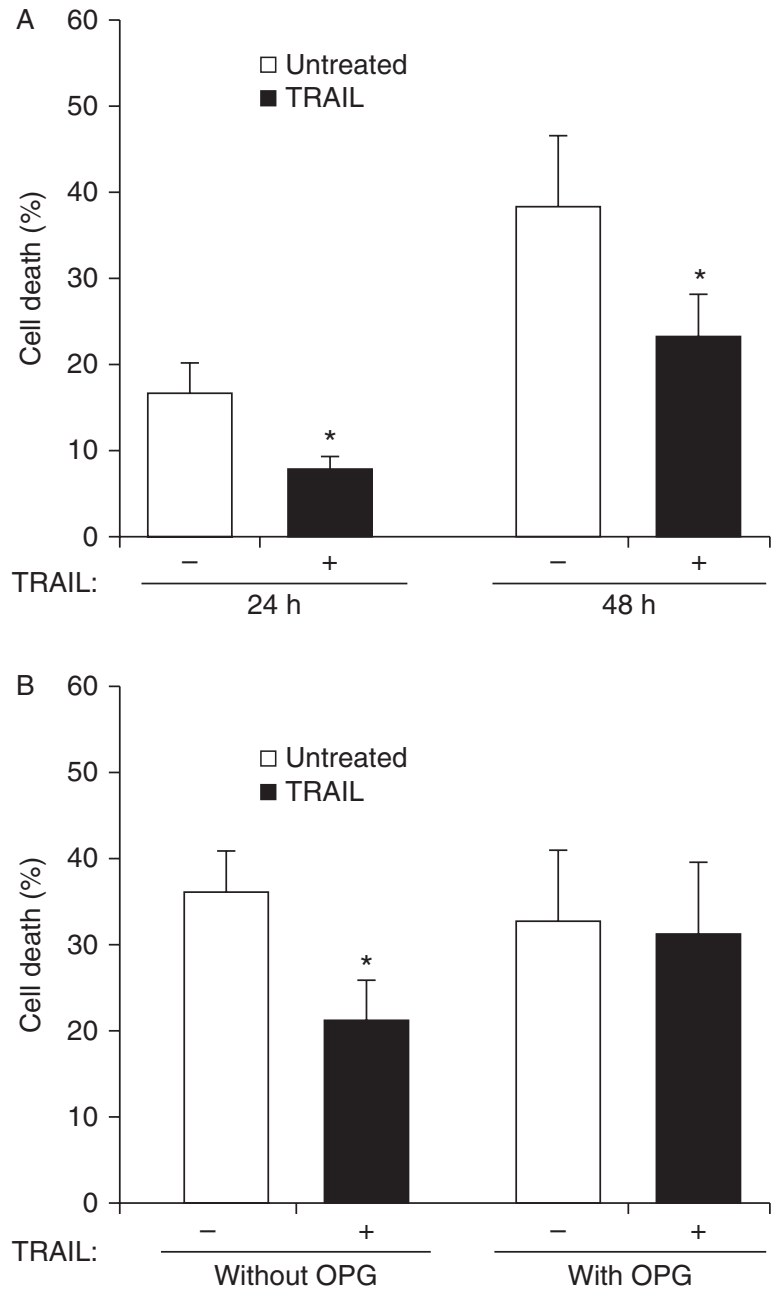

Figure 3 Effect of recombinant soluble TRAIL on spermatozoa vitality. Capacitated spermatozoa were exposed to recombinant TRAIL $(10 \mathrm{ng} / \mathrm{ml})$ and cell vitality/mortality was assessed by two different operators using the one-step eosin-nigrosin staining technique. (A) Cell vitality/mortality was assessed at the indicated time points; data from 12 independent experiments are reported as mean \pm s.D., $* P<0.05$ compared with untreated sample. (B) Cell vitality/mortality was assessed at $48 \mathrm{~h}$ after the indicated treatments with recombinant TRAIL $(10 \mathrm{ng} / \mathrm{ml})$ and OPG $(10 \mathrm{ng} / \mathrm{ml})$, used either alone or in combination. Data from three independent experiments are reported as mean \pm s.D., ${ }^{*} P<0.05$ compared with untreated sample.

samples, in which we observed that pre-incubation of TRAIL with an equal (1:1) concentration of OPG completely abrogated the pro-survival effect of TRAIL (Fig. 3B).

In parallel, we have investigated whether the in vitro treatment with recombinant TRAIL might impact the spermatozoa motility, by analyzing the distribution among the different WHO classes: (class A) rapid progressive motility, indicating sperm swimming with a progression velocity $>25 \mu \mathrm{m} / \mathrm{s}$; (class B) slow progressive motility, indicating sperm swimming with a progression velocity equal to $5-25 \mu \mathrm{m} / \mathrm{s}$; (class C) in situ motility; and (class D) no motility. As shown in Fig. 4, after $24 \mathrm{~h}$ of culture, the situation spontaneously changed drastically with respect to the motility pattern of the fresh capacitated samples (Table 2), in that percentage of classes $\mathrm{A}+\mathrm{B}$ spermatozoa significantly dropped in all samples, while classes $\mathrm{C}+\mathrm{D}$ significantly increased. Of note, in several samples the exposure to recombinant TRAIL resulted in an increment of the progressive motility (WHO classes $\mathrm{A}+\mathrm{B})$, counterbalanced by a parallel decrease in both $\mathrm{C}$ and $\mathrm{D}$ classes spermatozoa (Fig. 4).

\section{Discussion}

The role of seminal fluid in the regulation of human reproductive processes has been demonstrated in various studies (Eggert-Kruse et al. 2007, Robertson et al. 2009). Several cytokines secreted by the seminal vesicle and prostate gland modulate the response of various cell populations, including spermatozoa and white blood cells. It has also been demonstrated that seminal fluid affects the levels of cytokines and chemokines in human cervix, showing profound immunological effects (Sharkey et al. 2012). In this respect, the first major finding of the
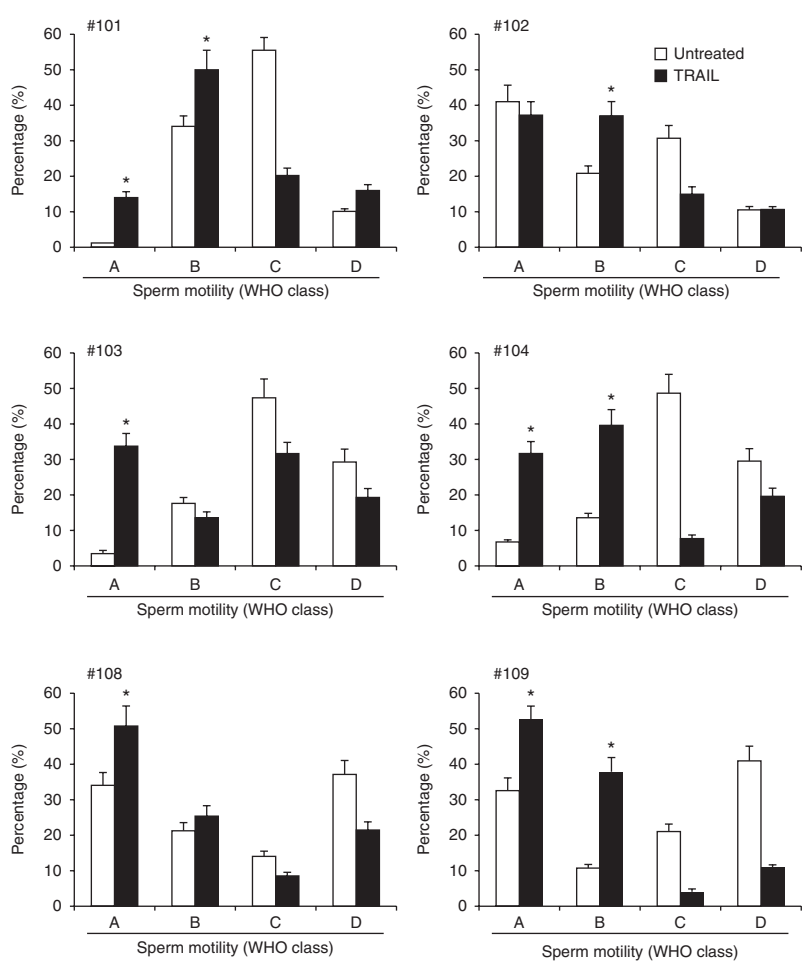

Figure 4 Effect of recombinant soluble TRAIL on spermatozoa mobility. Capacitated spermatozoa were exposed to recombinant TRAIL $(10 \mathrm{ng} / \mathrm{ml})$ before the assessment of sperm motility by two different operators. Results of six selected capacitated samples (out of 12 samples) are shown. Results are reported as mean \pm s.D. of experiments carried out in triplicate. ${ }^{*} P<0.05$ compared with untreated sample. 
present study was that TRAIL, a TNF family member, is present at high levels (median, $11621 \mathrm{pg} / \mathrm{ml}$ ) in seminal plasma. To appreciate how seminal plasma is enriched in TRAIL cytokine, it should be considered that the levels of TRAIL measured in human plasma, with the same commercial assay, are usually between 60 and $90 \mathrm{pg} / \mathrm{ml}$ (Secchiero et al. 2009, 2010, Volpato et al. 2011). Thus, seminal plasma contains $>2$ log higher concentrations of soluble TRAIL with respect to human plasma. Moreover, in this study, we have documented a variable expression of the surface TRAIL receptors in the spermatic cellular fraction. Although TRAIL/TRAILR system is generally considered to be a component of the cell anti-tumorigenic activity, its physiopathological role is broader than originally thought (Di Pietro \& Zauli 2004). In particular, the ability of TRAIL to suppress inflammatory reactions has been demonstrated in a variety of studies (Li et al. 2012, Marcuzzi et al. 2012, Keuper et al. 2013, Secchiero et al. 2013, Walczak 2013).

The second major finding of our study was that recombinant TRAIL, used at concentrations comparable to those found in seminal plasma $(10 \mathrm{ng} / \mathrm{ml})$, significantly increased the overall survival of capacitated spermatozoa. On the other hand, the survival/apoptotic rate of uncapacitated spermatozoa did not vary upon culture for $24-48 \mathrm{~h}$ in the absence or presence of recombinant TRAIL, probably due to the high spontaneous mortality/apoptosis. However, it is possible to speculate that the effect of TRAIL on capacitated spermatozoa could be due to the increase in surface TRAILR2 expression in capacitated spermatozoa as compared with pre-capacitated spermatozoa. The prosurvival/anti-apoptotic activity of TRAIL on spermatozoa was not totally unexpected, because we and other authors have previously demonstrated that the effect of TRAIL on survival/apoptosis is cell-type specific ( $\mathrm{Di}$ Pietro \& Zauli 2004). Indeed, while recombinant TRAIL induces apoptosis of most cancer cells in vitro, when added to primary normal cells can either exhibit no effects or it can activate intracellular pathways, such as ERK/MAPK, AKT, and NF- $\kappa \mathrm{B}$, which have been involved in the mediation of cell survival in different cell types (Secchiero et al. 2001, 2008, Milani et al. 2003, Zauli et al. 2005, 2008). In this respect, it is noteworthy that these pathways have been involved also in promoting the survival of spermatozoa in response to progesterone and other molecules (Rogers et al. 2008, Awda \& Buhr 2010, Espino et al. 2011, De Amicis et al. 2012, Gómez et al. 2012, Kim et al. 2012, Maheshwari et al. 2012, Sagare-Patil et al. 2013).

The third major finding of our study is that TRAIL, by promoting the cell survival, predominantly increased the capacitated spermatozoa of A+B motility classes, although this effect was not documentable in all the cases analyzed. Thus, although we have not a direct proof that the presence of TRAIL on seminal fluid has a direct impact on male semen fertility, the ability of TRAIL to prolong the survival of cells with forward progressive motility strongly suggest that TRAIL might play an important role as a trophic cytokines for capacitated spermatozoa. Taking into account that recombinant TRAIL is now in phase I and II clinical trials, the therapeutic potential of this cytokine in promoting spermatozoa survival/mobility should be further investigated.

In conclusion, our data indicated that soluble TRAIL is present in extremely high levels in seminal plasma and suggest that spermatozoa are the direct target of TRAIL, which acts by affecting the vitality of capacitated spermatozoa. It is particularly noteworthy that while our manuscript was under consideration for publication, a study performed in the TRAIL ${ }^{-1-}$ C57BL/6) model has demonstrated a fundamental role of TRAIL in preserving spermatogenesis, with adult TRAIL ${ }^{-1-}$ mice suffering a marked decline in the production of mature spermatozoa (Lin \& Richburg 2014). Therefore, our current data obtained in humans are in agreement with the study reported by Lin a\& Richburg, extending the protective activity of TRAIL to mature spermatozoa and further underlining the importance of TRAIL in the whole process of spermatogenesis.

\section{Declaration of interest}

The authors declare that there is no conflict of interest that could be perceived as prejudicing the impartiality of the research reported.

\section{Funding}

This study was funded by AIRC (Associazione Italiana per la Ricerca sul Cancro: Italian Association for Cancer Research) to G Zauli.

\section{Acknowledgements}

The authors acknowledge Dr Marilena Granzotto for cytofluorimetric technical analysis.

\section{References}

Awda BJ \& Buhr MM 2010 Extracellular signal-regulated kinases (ERKs) pathway and reactive oxygen species regulate tyrosine phosphorylation in capacitating boar spermatozoa. Biology of Reproduction 83 750-758. (doi:10.1095/biolreprod.109.082008)

Bernardi S, Milani D, Fabris B, Secchiero P \& Zauli G 2012 TRAIL as biomarker and potential therapeutic tool for cardiovascular diseases. Current Drug Targets 13 1215-1221. (doi:10.2174/138945012802002357)

BjörndahI L, Söderlund I \& Kvist U 2003 Evaluation of the one-step eosin-nigrosin staining technique for human sperm vitality assessment. Human Reproduction 18 813-816. (doi:10.1093/humrep/deg199)

Coureuil M, Ugolin N, Tavernier M, Chevillard S, Barroca V, Fouchet P \& Allemand I 2010 Puma and TRAIL/DR5 pathways control radiationinduced apoptosis in distinct populations of testicular progenitors. PLoS ONE 5 e12134. (doi:10.1371/journal.pone.0012134) 
De Amicis F, Santoro M, Guido C, Russo A \& Aquila S 2012 Epigallocatechin gallate affects survival and metabolism of human sperm. Molecular Nutrition \& Food Research 56 1655-1664. (doi:10. 1002/mnfr.201200190)

Di Pietro R \& Zauli G 2004 Emerging non-apoptotic functions of tumor necrosis factor-related apoptosis-inducing ligand (TRAIL)/Apo2L. Journal of Cellular Physiology 201 331-334. (doi:10.1002/jcp.20099)

Eggert-Kruse W, Kiefer I, Beck C, Demirakca T \& Strowitzki T 2007 Role for tumor necrosis factor $\alpha$ (TNF- $\alpha$ ) and interleukin 1- $\beta$ (IL-1 $\beta$ ) determination in seminal plasma during infertility investigation. Fertility and Sterility $\mathbf{8 7}$ 810-823. (doi:10.1016/j.fertnstert.2006.08.103)

Emery JG, McDonnell P, Burke MB, Deen KC, Lyn S, Silverman C, Dul E, Appelbaum ER, Eichman C, DiPrinzio R et al. 1998 Osteoprotegerin is a receptor for the cytotoxic ligand TRAIL. Journal of Biological Chemistry 273 14363-14367. (doi:10.1074/jbc.273.23.14363)

Espino J, Ortiz Á, Bejarano I, Lozano GM, Monllor F, García JF, Rodríguez AB \& Pariente JA 2011 Melatonin protects human spermatozoa from apoptosis via melatonin receptor- and extracellular signal-regulated kinase-mediated pathways. Fertility and Sterility 95 2290-2296. (doi:10.1016/j.fertnstert.2011.03.063)

Gómez M, Manzano A, Figueras A, Viñals F, Ventura F, Rosa JL, Bartrons R \& Navarro-Sabaté À 2012 Sertoli-secreted FGF-2 induces PFKFB4 isozyme expression in mouse spermatogenic cells by activation of the MEK/ERK/CREB pathway. American Journal of Physiology. Endocrinology and Metabolism 303 E695-E670. (doi:10.1152/ajpendo. $00381.2011)$

Grataroli R, Vindrieux D, Selva J, Felsenheld C, Ruffion A, Decaussin M \& Benahmed M 2004 Characterization of tumour necrosis factor- $\alpha$-related apoptosis-inducing ligand and its receptors in the adult human testis. Molecular Human Reproduction 10 123-128. (doi:10.1093/molehr/ gah016)

Keuper M, Wernstedt Asterholm I, Scherer PE, Westhoff MA, Möller P, Debatin KM, Strauss G, Wabitsch M \& Fischer-Posovszky P 2013 TRAIL (TNF-related apoptosis-inducing ligand) regulates adipocyte metabolism by caspase-mediated cleavage of PPAR $\gamma$. Cell Death \& Disease 4 e474. (doi:10.1038/cddis.2012.212)

Kim ST, Omurtag K \& Moley KH 2012 Decreased spermatogenesis, fertility, and altered Slc2A expression in Akt1 - / - and Akt2 - / - testes and sperm. Reproductive Sciences 19 31-42. (doi:10.1177/19337191 11424449)

Krause W \& Viethen G 1999 Quality assessment of computer-assisted semen analysis (CASA) in the andrology laboratory. Andrologia 31 125-129. (doi:10.1111/j.1439-0272.1999.tb01398.x)

Li J, Hsu HC, Yang P, Wu Q, Li H, Edgington LE, Bogyo M, Kimberly RP \& Mountz JD 2012 Treatment of arthritis by macrophage depletion and immunomodulation: testing an apoptosis-mediated therapy in a humanized death receptor mouse model. Arthritis and Rheumatism 64 1098-1109. (doi:10.1002/art.33423)

Lin YC \& Richburg JH 2014 Characterization of the role of tumor necrosis factor inducing ligand (TRAIL) in spermatogenesis through the evaluation of Trail gene-deficient mice. PLoS ONE 9 e93926. (doi:10.1371/journal. pone.0093926)

Maheshwari A, Misro MM, Aggarwal A \& Sharma RK 2012 N-acetylL-cysteine modulates multiple signaling pathways to rescue male germ cells from apoptosis induced by chronic hCG administration to rats. Apoptosis 17 551-565. (doi:10.1007/s10495-012-0703-8)

Marcuzzi A, Secchiero P, Crovella S \& Zauli G 2012 TRAIL administration down-modulated the acute systemic inflammatory response induced in a mouse model by muramyldipeptide or lipopolysaccharide. Cytokine $\mathbf{6 0}$ 43-46. (doi:10.1016/j.cyto.2012.06.001)

Milani D, Zauli G, Rimondi E, Celeghini C, Marmiroli S, Narducci P, Capitani S \& Secchiero P 2003 Tumour necrosis factor-related apoptosisinducing ligand sequentially activates pro-survival and pro-apoptotic pathways in SK-N-MC neuronal cells. Journal of Neurochemistry 86 126-135. (doi:10.1046/j.1471-4159.2003.01805.x)

Miraglia E, Lussiana C, Viarisio D, Racca C, Cipriani A, Gazzano E, Bosia A, Revelli A \& Ghigo D 2010 The pentose phosphate pathway plays an essential role in supporting human sperm capacitation. Fertility and Sterility 93 2437-2440. (doi:10.1016/j.fertnstert.2009.09.005)

Miyashita T, Kawakami A, Nakashima T, Yamasaki S, Tamai M, Tanaka F, Kamachi M, Ida H, Migita K, Origuchi T et al. 2004 Osteoprotegerin (OPG) acts as an endogenous decoy receptor in tumour necrosis factor- related apoptosis-inducing ligand (TRAIL)-mediated apoptosis of fibroblast-like synovial cells. Clinical and Experimental Immunology 137 430-436. (doi:10.1111/j.1365-2249.2004.02534.x)

Re MC, Zauli G, Gibellini D, Furlini G, Ramazzotti E, Monari P, Ranieri S, Capitani S \& La Placa M 1993 Uninfected haematopoietic progenitor $(\mathrm{CD} 34+)$ cells purified from the bone marrow of AIDS patients are committed to apoptotic cell death in culture. AIDS 7 1049-1055. (doi:10.1097/00002030-199308000-00004)

Rimondi E, Secchiero P, Quaroni A, Zerbinati C, Capitani S \& Zauli G 2006 Involvement of TRAIL/TRAIL-receptors in human intestinal cell differentiation. Journal of Cellular Physiology 206 647-654. (doi:10.1002/ jcp.20512)

Robertson SA, Guerin LR, Moldenhauer LM \& Hayball JD 2009 Activating $T$ regulatory cells for tolerance in early pregnancy - the contribution of seminal fluid. Journal of Reproductive Immunology 83 109-116. (doi:10.1016/j.jri.2009.08.003)

Rogers R, Ouellet G, Brown C, Moyer B, Rasoulpour T \& Hixon M 2008 Cross-talk between the Akt and NF- $\kappa$ B signaling pathways inhibits MEHP-induced germ cell apoptosis. Toxicological Sciences $\mathbf{1 0 6}$ 497-508. (doi:10.1093/toxsci/kfn186)

Sagare-Patil V, Vernekar M, Galvankar M \& Modi D 2013 Progesterone utilizes the PI3K-AKT pathway in human spermatozoa to regulate motility and hyperactivation but not acrosome reaction. Molecular and Cellular Endocrinology 374 82-91. (doi:10.1016/j.mce.2013.04.005)

Secchiero P \& Zauli G 2008 Tumor-necrosis-factor-related apoptosisinducing ligand and the regulation of hematopoiesis. Current Opinion in Hematology 15 42-48. (doi:10.1097/MOH.0b013e3282f15fa6)

Secchiero P, Gonelli A, Celeghini C, Mirandola P, Guidotti L, Visani G, Capitani S \& Zauli G 2001 Activation of the nitric oxide synthase pathway represents a key component of tumor necrosis factor-related apoptosis-inducing ligand-mediated cytotoxicity on hematologic malignancies. Blood 98 2220-2228. (doi:10.1182/blood.V98.7.2220)

Secchiero P, Melloni E, Corallini F, Beltrami AP, Alviano F, Milani D, D'Aurizio F, di lasio MG, Cesselli D, Bagnara GP et al. 2008 Tumor necrosis factor-related apoptosis-inducing ligand promotes migration of human bone marrow multipotent stroma cells. Stem Cells $\mathbf{2 6}$ 2955-2963. (doi:10.1634/stemcells.2008-0512)

Secchiero P, Corallini F, Ceconi C, Parrinello G, Volpato S, Ferrari R \& Zauli G 2009 Potential prognostic significance of decreased serum levels of TRAIL after acute myocardial infarction. PLOS ONE 4 e4442. (doi:10. 1371/journal.pone.0004442)

Secchiero P, Corallini F, Beltrami AP, Ceconi C, Bonasia V, Di Chiara A, Ferrari R \& Zauli G 2010 An imbalanced OPG/TRAIL ratio is associated to severe acute myocardial infarction. Atherosclerosis $210274-277$. (doi:10.1016/j.atherosclerosis.2009.11.005)

Secchiero P, Rimondi E, di lasio MG, Agnoletto C, Melloni E, Volpi I \& Zauli G 2013 C-reactive protein downregulates TRAIL expression in human peripheral monocytes via an Egr-1-dependent pathway. Clinical Cancer Research 19 1949-1959. (doi:10.1158/1078-0432. CCR-12-3027)

Sharkey DJ, Tremellen KP, Jasper MJ, Gemzell-Danielsson K \& Robertson SA 2012 Seminal fluid induces leukocyte recruitment and cytokine and chemokine mRNA expression in the human cervix after coitus. Journal of Immunology 188 2445-2454. (doi:10.4049/jimmunol. 1102736)

Vitale M, Zamai L, Falcieri E, Zauli G, Gobbi P, Santi S, Cinti C \& Weber G 1997 IMP dehydrogenase inhibitor, tiazofurin, induces apoptosis in K562 human erythroleukemia cells. Cytometry 30 61-66. (doi:10.1002/ (SICI)1097-0320(19970215)30:1 <61::AID-CYTO9>3.0.CO;2-I)

Volpato S, Ferrucci L, Secchiero P, Corallini F, Zuliani G, Fellin R, Guralnik JM, Bandinelli S \& Zauli G 2011 Association of tumor necrosis factor-related apoptosis-inducing ligand with total and cardiovascular mortality in older adults. Atherosclerosis 215 452-458. (doi:10.1016/ j.atherosclerosis.2010.11.004)

Walczak H 2013 Death receptor-ligand systems in cancer, cell death, and inflammation. Cold Spring Harbor Perspectives in Biology $\mathbf{5}$ a008698. (doi:10.1101/cshperspect.a008698)

Zauli G, La Placa M, Vignoli M, Re MC, Gibellini D, Furlini G, Milani D, Marchisio M, Mazzoni M \& Capitani S 1995 An autocrine loop of HIV type-1 Tat protein responsible for the improved survival/ proliferation capacity of permanently tat-transfected cells and required 
for optimal HIV-1 LTR transactivating activity. Journal of Acquired Immune Deficiency Syndromes and Human Retrovirology 10 306-316. (doi:10.1097/00042560-199511000-00002)

Zauli G, Sancilio S, Cataldi A, Sabatini N, Bosco D \& Di Pietro R 2005 $\mathrm{Pl}-3 \mathrm{~K} / \mathrm{Akt}$ and $\mathrm{NF}-\kappa \mathrm{B} / \mathrm{k} \mathrm{B} \alpha$ pathways are activated in Jurkat T cells in response to TRAIL treatment. Journal of Cellular Physiology 202 900-911. (doi:10.1002/jcp.20202)

Zauli G, Corallini F, Bossi F, Fischietti F, Durigotto P, Celeghini C, Tedesco F \& Secchiero P 2007 Osteoprotegerin increases leukocyte adhesion to endothelial cells both in vitro and in vivo. Blood 110 536-543. (doi:10.1182/blood-2007-01-068395)

Zauli G, Rimondi E, Stea S, Baruffaldi F, Stebel M, Zerbinati C, Corallini F \& Secchiero P 2008 TRAIL inhibits osteoclastic differentiation by counteracting RANKL-dependent p27Kip1 accumulation in pre-osteoclast precursors. Journal of Cellular Physiology 214 117-125. (doi:10. 1002/jcp.21165)

Zauli G, Melloni E, Capitani S \& Secchiero P 2009 Role of full-length osteoprotegerin in tumor cell biology. Cellular and Molecular Life Sciences 66 841-845. (doi:10.1007/s00018-008-8536-x)

Received 12 March 2014

First decision 9 April 2014

Revised manuscript received 12 May 2014

Accepted 13 May 2014 